\title{
Dietary Ether Lipid Incorporation into Tissue Plasmalogens of Humans and Rodents
}

\author{
Arun K. Das ${ }^{a}$, Ronald D. Holmes ${ }^{b}$, Golder N. Wilson ${ }^{c}$ and Amiya K. Hajra ${ }^{a, *}$

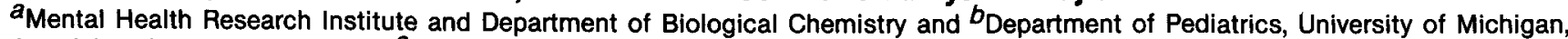 \\ Ann Arbor, Michigan 48104 and ${ }^{C}$ Department of Pediatrics, University of Texas, Southwestern Medical Center, Dallas, Texas 75235
}

Chronic feeding of 1-O-octadecyl-sn-glycerol (batyl alcohol) to patients suffering from congenital deficiency in tissue ether glycerolipids showed an increase in the plasmalogens content of their erythrocytes. However, nothing is known about the ether lipid content of other tissues in these patients. Feeding 1-O-heptadecyl-sn-glycerol to young rats showed that this uncommon ether lipid was incorporated to a high extent into the plasmalogens of all tissues except brain. Comparative studies with other precursors, such as 3-O-heptadecyl-sn-glycerol, heptadecanol and heptadecanoic acid, indicated a stereospecific incorporation of the dietary 1-O-alkyl-sn-glycerols into tissue plasmalogens without cleavage of the ether bond. Dietary ether lipids were also shown to be transferred from mothers to suckling rats, but not from pregnant rats to fetuses. The implication of these results to possible dietary ether lipid therapy for patients suffering from peroxisomal disorders is discussed.

Lipids 27, 401-405 (1992).

In mammals, 1-O-alkyl-sn-glycerols derived from dietary ether glycerolipids are absorbed without cleavage of the ether bond (1). This was first shown by Bergström and Blomstrand (2) who fed radioactive $1-O-\left[1^{\prime}-14 \mathrm{C}\right]$ hexadecylrac-glycerol (chimyl alcohol) to rats and showed that it was completely absorbed by the intestine. About half of the radioactive lipids recovered from the lymphatic fluid were shown to be either chimyl alcohol or its esterified derivatives, and the rest of the radioactivity was recovered in palmitic acid. Using human subjects, a similar finding was reported by Blomstrand and Ahrens (3). Apparently after absorption, all of the unnatural isomer (sn-3-alkyl) and part of the natural (sn-1-alkyl) isomer were oxidized in the intestinal mucosal cells to form palmitic acid (4). This was demonstrated later by Paltauf (5) who administered labeled natural (1-O-hexadecyl-sn-glycerol) and unnatural (3-O-hexadecyl-sn-glycerol) glycerol ether isomers to rats and showed that only the natural isomer was incorporated into intestinal mucosal lipids. Paltauf also showed that the exogenous alkylglycerol was further metabolized to plasmalogens in intestinal mucosal cells (5). Mangold (1) and Bandi et al. (6) reported similar findings by feeding rats glycerol ethers which contained unnatural $(18: 2,18: 3)$ alkyl chains. These works have found that dietary ether glycerolipids are not only incorporated into membrane ether lipids of intestinal mucosal cells, but a small

*To whom correspondence should be addressed at the University of Michigan, Neuroscience Laboratory, 1103 E. Huron St., Ann Arbor, MI 48104-1687.

Abbreviations: DHAP, dihydroxyacetone phosphate; DHAPAT, dihydroxyacetone phosphate acyltransferase; GPC, glycerophosphocholine; GPE, glycerophosphoethanolamine. portion is also transported to liver and incorporated into plasmalogens in that tissue (6). Reichwald and Mangold (7) fed rats 1-alkyl glycerols having unnatural, odd-numbered and polyunsaturated alkyl groups (13:0, 15:0, 17:0, 19:0, 19:1 and 19:2) and studied their incorporation into lipids of intestinal mucosal cells. They found that although all of these glycerol ethers are incorporated into alkylacyl-glycerophosphoethanolamine (GPE) and alkylacyl-glycerophosphocholine (GPC), only the glycerol ethers containing 17:0, 19:0 and 19:1 chain lengths are converted into the corresponding ethanolamine and choline plasmalogens. Weber (8) showed that dietary 1-O-dodecylrac-glycerol is incorporated into the alkylglycerolipids, but not into the plasmalogens, of all tissues in mice.

These studies from different laboratories established that mammals can utilize dietary glycerol ethers to make membrane ether lipids in different tissues. In recent years, the possible incorporation of exogenous ether lipids into tissue plasmalogens has assumed clinical significance because of the discovery of a number of genetic diseases involving ether lipid deficiency (9-11).

Heymans et al. (12) first reported the almost complete absence of ether lipids in the tissues of patients suffering from Zellweger cerebrohepatorenal syndrome. This deficiency of ether lipids is due to the absence of peroxisomes in the tissues of these patients (13). Peroxisomes have been shown to be the site for synthesis of ether lipids (14). It has been demonstrated that the activity of dihydroxyacetone phosphate acyltransferase (DHAPAT), which catalyzes the biosynthesis of the precursor of ether lipids, acyl dihydroxyacetone phosphate (acyl DHAP) (Fig. 1), is very low in the tissues of Zellweger patients (15-17). The activity of the enzyme catalyzing the synthesis of the ether bond, i.e., alkyl DHAP synthase (Fig. 1), is also somewhat low in these patients $(15,17,18)$. The deficiency of these peroxisomal enzymes, required for the de novo biosynthesis of the ether bond in ether glycerolipids, results in a decreased amount of plasmalogens in the tissues of Zellweger patients. The same enzymes have also been shown to be deficient in a number of other genetic diseases involving peroxisomal disorders, such as neonatal adrenoleukodystrophy (19), infantile Refsum disease (20), rhizomelic chrondrodysplasia punctata (9) and others (21). On the other hand, the activity of the key enzyme necessary for the utilization of exogenous 1-O-alkyl-sn-glycerol, i.e., alkyl glycerol kinase (Fig. 1), was shown not to be deficient in these patients (15). Considering that the enzymes that catalyze the conversion of 1-alkyl-sn-glycerol-3-phosphate to membrane ether glycerolipids and plasmalogens are localized not in peroxisomes $(14,22)$ but in the endoplasmic reticulum (Fig. $1)$, it is expected that exogenous alkyl glycerols might be incorporated into tissue ether lipids in these patients. This has been shown to be the case in cultured skin fibroblasts 


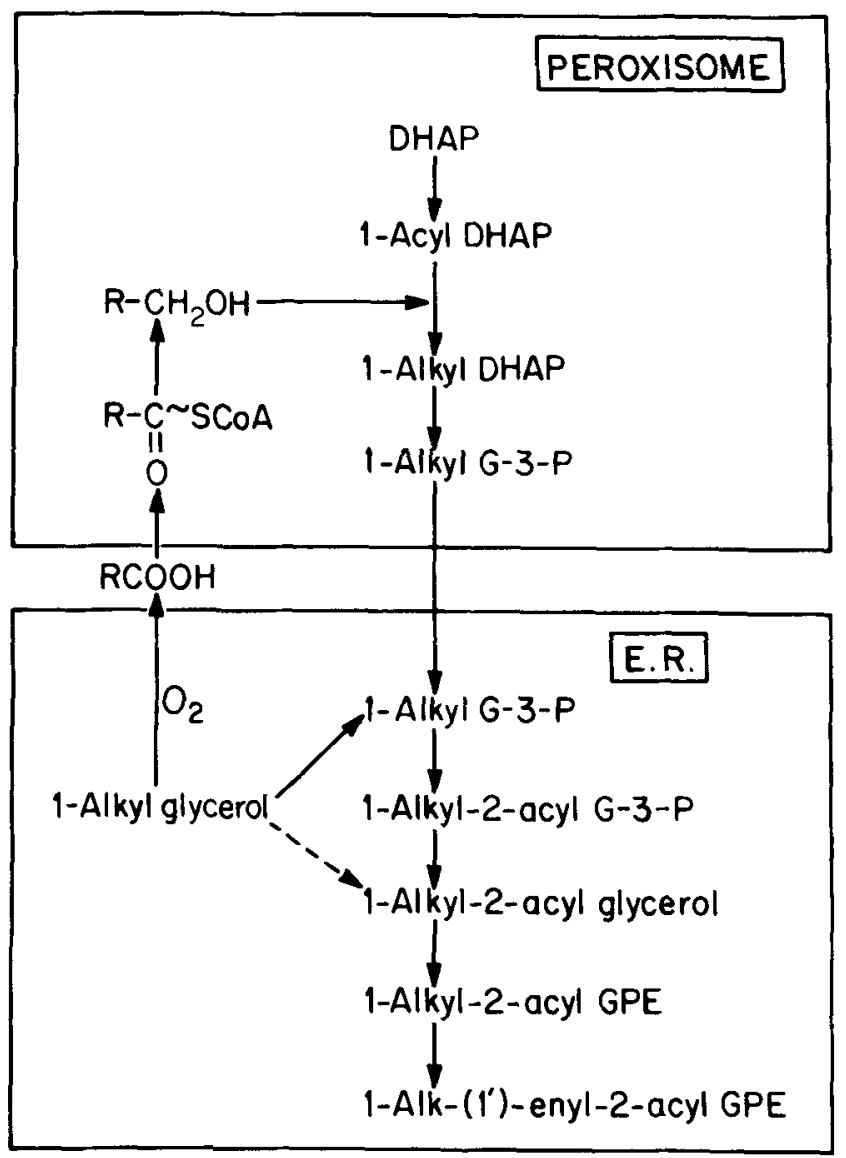

FIG. 1. Subcellular compartmentation of the enzymatic reactions leading to the biosynthesis of alkyl glycerol ethers and plasmalogens.

obtained from Zellweger patients where the labeled alkyl glycerols, added to the culture medium, are incorporated into cellular plasmalogens, but the labeled long-chain alcohols are not $(23,24)$.

\section{ORAL ETHER LIPID THERAPY IN PATIENTS WITH ETHER LIPID DEFICIENCY}

The findings summarized above led us to hypothesize that oral administration of ether lipids to patients with peroxisomal disorders might rectify the tissue ether lipid deficiency $(15,25)$. Our group has given several patients with peroxisomal disorders an ether lipid suspension containing $400 \mathrm{mg} \mathrm{1-O-octadecyl-sn-glycerol} \mathrm{(batyl} \mathrm{alcohol)} \mathrm{and}$ $200 \mathrm{mg}$ sodium deoxycholate in $100 \mathrm{~mL}$ of water. Each patient received 5-10 mg/kg daily of batyl alcohol (25). Case histories for three patients are described below.

Patient 1. NR was born on May 26, 1983, following a full-term gestation. Birth weight was $3.13 \mathrm{~kg}(50 \%)$, length $54 \mathrm{~cm}(95 \%)$ and head circumference $34.5 \mathrm{~cm}(50 \%)$. He was evaluated at 7 weeks of age because of failure to thrive and was noted to have hypotonia and enlarged anterior and posterior fontanels with diastasis of the sagittal suture. Hepatomegaly was present at age 4 months and serum aminotransferase levels were elevated. Percutaneous liver biopsy showed abnormal lobular architecture with fibrous septa extending from portal tracts into the surrounding parenchyma. By 8 months of age he could not visually fix on objects and had wandering nystagmus. Ophthalmologic evaluation revealed bilateral punctate cortical and plaque-like nuclear changes, optic atrophy and retinal degeneration.

A skin biopsy was performed at 1 year of age and fibroblasts were cultured, harvested and assayed for the activity of the peroxisomal membrane enzyme, dihydroxyacetone phosphate acyltransferase (DHAPAT). The activity was $30 \%$ of control. Plasma levels of $\mathrm{C}_{26}$ very longchain fatty acids and the ratio of $\mathrm{C}_{26} / \mathrm{C}_{22}$ assayed at 20 months of age were elevated in a pattern diagnostic of neonatal adrenoleukodystrophy.

Dietary supplementation with batyl alcohol was started in August, 1984, and continued through May, 1987. The child is currently enrolled in special education classes with a level of development equivalent to a young toddler. $\mathrm{He}$ has not yet developed clinical or laboratory evidence of adrenal insufficiency (normal cortrosyn stimulation test at age $81 / 2$ years).

Patient 2. EZ was born at 40 weeks gestation by emergency cesarean section on September 28, 1982, because of fetal distress and maternal hypertension. Birth weight was $2.5 \mathrm{~kg}$ and length $53 \mathrm{~cm}$. At 6 months of age she was evaluated for failure to thrive, hypotonia and hepatomegaly. A liver biopsy was performed and sections of the liver showed bridging fibrosis and paucity of intrahepatic bile ducts without cholestasis.

She was re-evaluated at age 2 years because of failure to thrive, delayed development, seizures, hypotonia and persistent hepatomegaly with elevated levels of serum aminotransferases. Her facies was not dysmorphic, although there was a high-arched palate. Because of these findings, fibroblasts cultured from a skin biopsy were assayed for DHAPAT. The activity of fibroblast DHAPAT was $0.125 \mathrm{nmol} / \mathrm{min} / \mathrm{mg}$ protein (control value $=0.2 \mathrm{nmol} /$ $\mathrm{min} / \mathrm{mg}$ protein). The reference non-peroxisomal enzyme glycerol-3-phosphate acyltransferase had values of 0.89 $\mathrm{nmol} / \mathrm{min} / \mathrm{mg}$ (control $=0.81 \mathrm{nmol} / \mathrm{min} / \mathrm{mg}$ protein). Erythrocyte plasmalogen content was assayed at age 3 years and was abnormal (Table 1).

She was given a suspension of batyl alcohol from July, 1985, until February, 1989. There were no adverse effects.

\section{TABLE 1}

Erythrocyte Ethanolamine Plasmalogen Concentration Before and After Administration of Batyl Alcohol ${ }^{a}$

\begin{tabular}{lccc}
\hline & \multicolumn{3}{c}{$\begin{array}{c}\text { Ethanolamine plasmalogen } \\
\text { (\% of total phospholipids) }\end{array}$} \\
\cline { 2 - 4 } & $\begin{array}{c}\text { Patient } \\
\text { no. 1 }\end{array}$ & $\begin{array}{c}\text { Patient } \\
\text { no. 2 }\end{array}$ & $\begin{array}{c}\text { Patient } \\
\text { no. 3 }\end{array}$ \\
\hline Pretreatment value & 4.0 & 1.3 & 4.1 \\
Post-treatment value & 10.6 & 11.2 & 7.5 \\
\hline
\end{tabular}

a Erythrocyte phospholipid composition was analyzed by two-dimensional thin-layer chromatography as previously described (30). The percentage of ethanolamine plasmalogen is shown here. Most of the other phospholipid percentages were the same as normal except for phosphatidylethanolamine, which was increased. The ethanolamine plasmalogen contents of normal subjects varies from $10 \%$ to $15 \%$ of the total phospholipids. For further details, see text and ref. 25. 
She is presently attending a school for developmentally handicapped children and is beginning to walk. There is marked speech delay.

Patient 3. CB was born on January 14, 1986, following full-term gestation. Hypotonia and strabisimus were present at birth. He was evaluated at age 4 months because of failure to thrive, and was noted to have a dysmorphic facies and hepatomegaly. Plasma very long chain fatty acid levels were elevated and the activity of fibroblast DHAPAT was determined to be $40 \%$ of control value. These findings supported the diagnosis of neonatal adrenoleukodystrophy.

A cortrysn stimulation test was performed in March, 1988. The result was abnormal and hydrocortisone replacement therapy was started. In October, 1988, he developed a diffuse, erythematous skin rash, associated with elevation of plasma phytanic acid.

An oral suspension of batyl alcohol was prescribed in October, 1986, and continued to January, 1989. The erythrocyte plasmalogen content was measured before and after treatment with batyl alcohol. The results are summarized in Table 1.

At the present time, he is attending a special education program for hearing-impaired and vision-impaired children. He has marked developmental delay and is not yet able to walk.

The results presented in Table 1 show that erythrocyte plasmalogen levels are increased in these patients after oral feeding of batyl alcohol for a few months. In one patient, who was under oral batyl alcohol therapy for three months, the chain length distribution of the alk-1'-enyl moiety of ethanolamine plasmalogens from erythrocytes was analyzed. An increase in the $18: 0$ moiety $168 \%$ vs. $50 \%$ in normal controls) was observed, suggesting that the dietary batyl alcohol was absorbed, metabolized and incorporated into the erythrocyte plasmalogens of this patient.

Administration of oral ether lipids represents a potential treatment for patients with peroxisomal disorders. Although none of the patients reported in this series have the severe Zellweger phenotype associated with multiple peroxisomal enzyme deficiency, they all share clinical features typically seen in patients with peroxisomal dysfunction: failure to thrive, delayed development, hypotonia, facial dysmorphia and hepatomegaly. Each of these patients has a documented defect of plasmalogen synthesis and oxidation of fatty acids. These infants have exhibited subjective improvement of nutritional status, liver function, retinal pigmentation and motor tone and repletion of deficient red cell ethanolamine plasmalogens since receiving batyl alcohol. However, it is not possible to separate these changes from the natural history of untreated disease. Wanders et al. (26) have documented increasing plasmalogen levels in Zellweger's syndrome as a function of age. This may be due to the intake of natural alkylglycerols present in food. Horrocks (27) estimated that the average adult consumes 10-100 mg of batyl alcohol each day.

\section{INCORPORATION OF DIETARY ALKYLGLYCEROL INTO PLASMALOGENS OF DIFFERENT TISSUES IN RATS}

The increase in the plasmalogen level in erythrocytes of patients after feeding batyl alcohol indicated that dietary ether lipids may also be incorporated into different tissues of these patients. However, a direct demonstration of such an increase in tissues of these patients was not feasible. There are also no reports of incorporation of dietary ether lipids into plasmalogens of different tissues of animals except in intestinal mucosa $(1,5)$ and, to a small extent, in liver of rats (6). By contrast, there are reports of nonincorporation of exogenous tracer doses of labeled ether lipids into tissue plasmalogens of animals $(28,29)$. Therefore, to determine whether dietary ether lipids are utilized to synthesize membrane ether lipids of different tissues, we administered 1. $O$-heptadecyl-sn-glycerol to young rats. The odd chain heptadecyl moiety is practically absent in normal ether glycerolipids, but it is very similar to the alkyl and alk-1'-enyl groups of tissue lipids that are mainly composed of 16:0, 18:0 and 18:1 chains. By feeding rats (19-d-old) 1-O-heptadecyl-rac-glycerol (1-2\% mixed with food) for 5 to 10 days, we found a very high (46-60\%) incorporation of the $\mathrm{C}_{17}$-moiety at the $\mathrm{C}-1$ position of ethanolamine plasmalogens in most tissues of these rats (30). However, the total plasmalogen content of the tissues did not increase. These results show that dietary alkyl glycerol ethers are not only absorbed and converted to plasmalogens in intestinal tissue, but are also transported to other organs where they are metabolized and deposited as membrane plasmalogens. The incorporation of the exogenous ether lipids into tissue plasmalogens is quite high, much higher than reported previously (7). This is probably due to our use of young growing rats for these experiments, as opposed to the use of adult rats by other authors. Recently, Blank et al. (31) reported a similar observation. These authors fed 1-O-alkyl-2,3-diacetyl-snglycerol, containing 65\% 18:1 and 17\% 16:1 alkyl groups, to rats, and found an increase in the 18:1 group of alkyl and alk-1'-enyl side chains of ether phosphoglycerides in liver, kidney and lung. There was an increase in the alkyl glycerol ether content but, as we have found, no change in the plasmalogens content of these tissues (31). We further investigated the mode of incorporation of dietary ether lipids into tissue lipids by feeding different precursors of ether lipids, such as heptadecanoic acid, heptadecanol, 1-O-heptadecyl-sn-glycerol and 3-O-heptadecylsn-glycerol, to young rats. The results are summarized in Figure 2. It is seen that 1-O-heptadecyl-sn-glycerol is the best precursor, whereas its unnatural optical isomer, 3-Oheptadecyl-sn-glycerol, is incorporated only to a very small extent. Apparently, the $s n-3$ isomer is oxidized to heptadecanoic acid (the mono-oxygenase oxidizing the ether bond is non-stereospecific, see ref. 32) which is further utilized. This conclusion is supported by the higher percentage of incorporation of heptadecanol and heptadecanoic acid than of the $s n-3$ isomer (Fig. 2). The relative incorporation rates, i.e. 1-O-heptadecyl-sn-glycerol $>$ heptadecanol $>$ heptadecanoic acid $>3$-O-heptadecylsn-glycerol (Fig. 2), suggest that the pathway shown (Fig. 1) for the incorporation of different precursors into ether lipids is operative in all tissues. These results and results from other laboratories $(7,8,31)$ indicate that most exogenous long chain ether lipids incorporate into alkylglycerol ether lipids, but the subsequent conversion to plasmalogens occurs only in lipids containing alkyl chain lengths between $\mathrm{C}_{15}$ and $\mathrm{C}_{19}$ (saturated or monounsaturated). Thus, it appears that, physiologically, the 1 '-alkenyl chain lengths are restricted to a narrow range, 

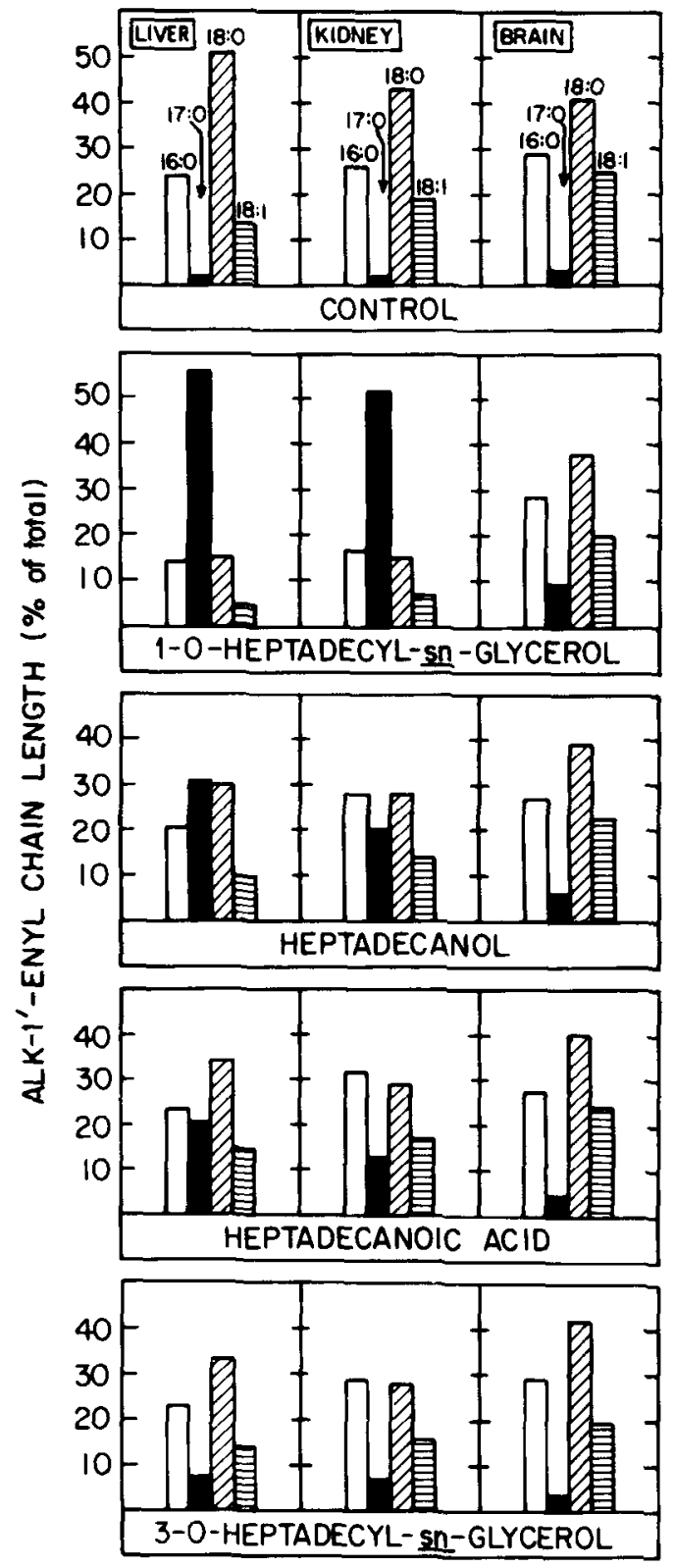

FIG. 2. Incorporation of dietary 1-0-heptadecyl-sn-glycerol, heptadecanol, heptadecanoic acid and 3-O-heptadecyl-8n-glycerol into ethanolamine plasmalogen of the tissues of 19-day-old rats. Rats were fed either powdered food mixed with the indicated compounds $(1 \%$, w/w) or only the powdered food alone (control group) for 5 days, and then the chain length distributions of the alk-1'enyl group at C-1 position of ethanolamine plasmalogen of liver, kidney and brain were analyzed, as described previously (30).

not only by the specificity of the acyl-CoA reductase (33), but also by the chain length specificity of the dehydrogenase which converts 1-O-alkyl phosphoglycerides to $1-O$ [1'-alkenyl]phosphoglycerides (34). The nature of the ether lipids transported from intestine to liver or to other organs is a matter of speculation. However, it is plausible that esterified alkylglycerols (ether analogs of triglycerides) are transported from the intestine (2-4) and liver to other organs and that these alkylglycerol derivatives are utilized after partial or complete hydrolysis of the ester bonds. Neutral lipids, whose chromatographic migration rate is similar to alkyldiacylglycerol, were found to be present in the liver and kidney of animals who were chronically fed heptadecylglycerol (Das, A.K., and Hajra, A.K., unpublished observation; see also ref. 31 ).

The results of our animal feeding experiment show that dietary ether glycerolipids are effectively utilized to synthesize membrane ether lipids in most tissues. However, in brain the incorporation of the exogenous ether lipid precursor was always very low (ref. 30 and Fig. 2). This could possibly be due to either a low rate of ether lipid synthesis in brain after myelination is complete, or to the fact that the ether lipid precursors cannot cross the bloodbrain barrier. To resolve this question, ether lipids should be fed to rats before the start of myelination $(<10 \mathrm{~d}$ old $)$. It is difficult, however, to feed large amounts of ether lipids to these suckling rats. Therefore, we fed pregnant female rats powdered food containing $0.5 \%(w / w) ~ 1-O-$ heptadecyl-sn-glycerol on the fifth day before delivery, and continued until the fourteenth day after the pups were born. The babies were allowed to suck the mother's milk which, when analyzed, was found to contain 1-O-alkyl2,3-diacyl-sn-glycerols. When the tissues of the babies (14-d-old) were analyzed, $28 \%$ of liver and $17 \%$ of kidney alk-1'enyl groups of ethanolamine plasmalogen were found to contain the 17:0 moiety. These values were lower than those obtained by direct feeding of heptadecylglycerol (30). In brain, a low $(2 \%)$ incorporation of 17:0 in the alkenyl chain was also observed. It is not clear whether this low incorporation into brain lipid is due to the blood-brain barrier or to the lower consumption of heptadecylglycerol by the babies. In another experiment, we found no incorporation of the 17:0 moiety in the tissues of newborn babies whose mothers had been fed 1-O-heptadecylglycerol for 20 days during pregnancy, though the mothers' tissues contained a high percentage $(40-60 \%)$ of the heptadec-1'enyl moiety in the ethanolamine plasmalogens. This indicates that ether lipids are not transported across the placenta from mother to fetus.

From these results, it is evident that dietary ether lipids can be directly utilized by mammals to synthesize membrane alkyl glycerolipids and plasmalogens in most tissues. Although the utilization of the exogenous ether lipids for the formation of brain plasmalogens is somewhat questionable, there was a low but reproducible incorporation of 1-O-heptadecyl-sn-glycerol into brain ether lipids (ref. 30, Fig. 2). It is possible that the exogenous ether lipids would be more effectively utilized if there were no endogenous formation of ether lipids. Increasing the dose might result in higher incorporation of ether lipids in all tissues of the patients. Oral ether lipid therapy may never relieve all of the syndrome associated with these diseases because of multiple peroxisomal enzyme defects $(10,22)$. But, in mild cases, where the main problem is the deficiency of ether lipids, oral ether lipid therapy may prove helpful. Alkyl glycerols are fairly innocuous compounds which have been shown to be nontoxic even at high doses (1), and no harmful effects are expected when these compounds are chronically administered to the patients. Oral ether lipid therapy should be attempted to alleviate some of the symptoms associated with these peroxisomal disorders. 


\section{ACKNOWLEDGMENT}

This work was supported by NIH grants NS 15747 and NS 08841 .

\section{REFERENCES}

1. Mangold, H.K. (1983) in Ether Lipids: Biochemical and Biomedical Aspects (Mangold, H.K., and Paltauf, F., eds.) pp. 231-238, Academic Press, New York.

2. Bergström, S., and Blomstrand, R. (1956) Acta Physiol. Scand. $38,166-172$.

3. Blomstrand, R., and Ahrens, Jr., E.H. (1959) Proc. Soc. Exp. Biol. Med. 100, 802-805.

4. Blomstrand, R. (1959) Proc. Soc. Exp. Biol. Med. 102, 662-665.

5. Paltauf, F. (1971) Biochim. Biophys. Acta 239, 38-46.

6. Bandi, Z.L., Mangold, H.K., Holmer, G., and Aaes-Jorgensen, E. (1971) FEBS Lett. 12, 217-220.

7. Reichwald, I., and Mangold, H.K. (1977) Nutr. Metab. Suppl. 21, 198-201.

8. Weber, N. (1985) J. Lipid Res. 26, 1412-1420.

9. Schutgens, R.B.H., Heymans, H.S.A., Wanders, R.J.A., van den Bosch, H., and Tager, J.M. (1986) Eur. J. Pediatr. 144, 430-444.

10. Moser, H.W. (1986) J. Pediatr. 108, 89-91.

11. Wilson, G.N., Holmes, R.D., and Hajra, A.K. (1988) Am. J. Med. Genet. 30, 771-792.

12. Heymans, H.S.A., Schutgens, R.B.H., Tan, R., van den Bosch, H., and Borst, P. (1983) Nature 306, 69-70.

13. Goldfischer, S., Moore, C.L., Johnson, A.B., Spira, A.J., Valsamis, M.P., Wisniewski, H.K., Ritsch, R.H., Norton, W.T., Rapin, I., and Gartner, L.M. (1973) Science 182, 62-64.

14. Hajra, A.K., and Bishop, J.E. (1982) Ann. N.Y. Acad. Sci. 386, 170-181.

15. Datta, N.S., Wilson, G.N., and Hajra, A.K. (1984) N. Engl. J. Med. 311, 1080-1083.

16. Schutgens, R.B.H., Romeyn, G.J., Wanders, R.J.A., van den Bosch, H., Schrakamp, G., and Heymans, H.S.A. (1984) Biochem. Biophys. Res. Commun. 120, 179-184.

17. Webber, K.O., Datta, N.S., and Hajra, A.K. (1987) Arch Biochem. Biophys. 254, 611-620.

18. Schrakamp, G., Rosenbloom, C.F.P., Schutgens, R.B.H., Wanders, R.J.A., Heymans, H.S.A., Tager, J.M., and van den Bosch, H. (1985) J. Lipid Res. 26, 867-873.
19. Kelley, R.I., Datta, N.S., Dobyns, W.B., Hajra, A.K., Moser, A.B., Noetzel, M.J., Zackai, E.H., and Moser, H.W. (1986) Am. J. Med. Genet. 23, 869-901.

20. Poulos, A., Pollard, A.C., Mitchel, J.D., Wise, D., and Mortimer, G. (1984) Arch. Dis. Child. 59, 222-229.

21. Hajra, A.K., Das, A.K., Webber, K.O., Holmes, R.G., and Wilson, G.N. (1988) in Lipid Storage Disorders: Genetic Diseases Affecting Peroxisomal Lipid Biosynthesis (Salvayre, R., Douste-Blasy, L., and Gatt, S., eds.) pp. 369-380, Plenum Publishing Corporation, New York.

22. Hajra, A.K., Horie, S., and Webber, K.O. (1988) in Biological Membranes: Aberrations in Membrane Structures and Function (Karnovsky, M.L., Leaf, A., and Bolis, L.C., eds.), pp. 99-116, Alan R. Liss, New York.

23. Schrakamp, G., Schutgens, R.B.H., Wanders, R.J.A., Heymans, H.S.A., Tager, J.M., and van den Bosch, H. (1985) Biochim. Biophys. Acta 833, 170-174.

24. Roscher, A., Molzer, B., Bernheimer, H., Stockler, S., Mutz, I., and Paltauf, F. (1985) Pediatr. Res. 19, 930-933.

25. Wilson, G.N., Holmes, R.G., Custer, J., Lipkowitz, J.L., Stover, J., Datta, N.S., and Hajra, A.K. (1986) Am. J. Med. Genet. 24, 69-82.

26. Wanders, R.J.A., Purvis, Y.R., Heymans, H.S.A., Bakkeren J.A.J.M., Parmentier, G.G., Eldere, J.V., Eyssen, H., van den Bosch, H., Tager, J.M., and Schutgens, R.B.H. (1986) J. Inher. Metab. Dis. 9, 335-342.

27. Horrocks, L.A. (1972) in Ether Lipids: Chemistry and Biology (Snyder, F., ed.) pp. 177-272, Academic Press, New York.

28. Snyder, F., and Pfleger, R.C. (1966) Lipids 1, 328-334.

29. Ahrne, L., Bjorck, L., and Claesson, O. (1982) Ann. Nutr. Metab. $26,162-170$.

30. Das, A.K, and Hajra, A.K. (1988) FEBS Lett. 227, 187-190.

31. Blank, M.L., Cress, F.A., Smith, Z.L., and Snyder, F. (1991) Lipids $26,166-169$.

32. Tietz, A., Lindberge, M., and Kennedy, E.P. (1964) J. Biol Chem. 239, 4081-4090.

33. Bishop, J.E., and Hajra, A.K. (1981) J. Biol. Chem. 256, 9542-9550.

34. Paltauf, F. (1983) in Ether Lipids: Biochemical and Biomedical Aspects (Mangold, H.K., and Paltauf, F., eds.) pp. 107-128, Academic Press, New York.

[Received June 26, 1991; Revision accepted March 28, 1992] 